\title{
DYNAMIC OUTPUT FEEDBACK CONTROL FOR SAMPLED-DATA SYSTEM WITH ACTUATOR SATURATION USING QUANTIZED MEASUREMENTS
}

\author{
Liuwen $\mathrm{Li}^{1}$, Wenlin $\mathrm{Zou}^{2}$ and Shumin $\mathrm{Fei}^{1}$ \\ ${ }^{1}$ School of Automation, Southeast University, Nanjing, China \\ ${ }^{2}$ School of Computer Science and Engineering, Nanjing University of Science \\ and Technology, Nanjing, China
}

\begin{abstract}
This paper is focused on the dynamic output feedback control of sampled-data system with actuator saturation using quantized measurements. The output signals are quantized before they are transmitted to dynamic output-feedback controller. An augmented system is constructed through a closed-loop system, which is a model with an interval time-varying delay and nonlinear items. Using discontinuous Lyapunov function and linear matrix inequality approach, the criterion of asymptotical stability is obtained; furthermore, on the basis of the above stability condition, we design a dynamic output-feedback controller. Lastly, the effective of the proposed method is proved by a numerical example.
\end{abstract}

\section{KEYWORDS}

Discontinuous Lyapunov function, Dynamic output-feedback control, Sampled-data control systems, Saturations, quantization.

\section{INTRODUCTION}

For several decades, the stability of control system has been researched by many researchers because of its wide usage in many fields. In recent years, more and more control problems turn to digital controllers to solve. The key point of this control method is at discrete time but not continuous time of the state variable samples. Therefore, sampled-data control systems $[15,16,18$, 19] have attracted much attention. Three main approaches have been used to research the sampled-data control of linear systems. The first approach is discrete-time models [1,20]. The second one is impulsive model [3]. And the third is time-delay model which is via constructing Lyapunov-Razumikhin functions or Lyapunov-Krasovskii functional and using LMIs analyze the stability and design the controller of sampled-data control systems [2, 4, 15, 22, 23].

Saturation is very common in practical application because of the technological or safety constraints. Considering the importance of the saturation, researchers have studied saturation with the problem of filtering $[5-9,20]$ and the problem of control [6-8, 10, 21]. Just as saturation, control using quantized feedback has been a hot research topic for many years. Kalman studied the effect of quantization in a sampled data system in 1956 and presented that the feedback system would exhibit limit cycles and chaotic behaviour if a stabilizing controller is quantized using a Natarajan Meghanathan et al. (Eds) : ACSIT, SIPM, FCST, CoNeCo, CMIT - 2017 
finite-alphabet. [25] studied a number of quantized feedback design problems for linear systems and the main discovery is that the classical sector bound method is non conservative for studying the design problems. The problem of quantized state-feedback control has been investigated through a quantization dependent approach in [26] and new stability and stabilization conditions have been given. Quantization has also emerged in many literatures, such as in[8], [24], [27]. In this paper, we develop the static output-feedback in [15] to dynamic output-feedback control system under saturation and quantization constraint.

This paper concentrates on asymptotical stability of the sampled data control system under saturation and quantization constraint, controlled by dynamic output-feedback. Our aim is to analyze the performance of the closed loop system under above constraint. Firstly, a model of sampled-data system is constructed. There for, based on the discontinuous Lyapunov function and employing linear matrix inequality approach, sufficient conditions for system stability are obtained. Then, a dynamic output-feedback controller is designed on the basis of above stability. Finally, the practical utility of this method is proved by a numerical example.

The paper is organized as follows. Section 2 presents some useful lemmas and definition. Section 3 presents the system and the dynamic output-feedback controller we study. Section 4gives sufficient condition of system stability and dynamic output-feedback controller through linear matrix inequality approach. Section 5 illustrates the obtained results by a numerical example, which is followed by the conclusion in Section 6.

\section{LEMMAS AND DEFINITIONS}

In order to describe the system more clearly, we will give the following definitions and lemmas.

Lemma 1[12]The saturation function $\delta(u(t))$ can be decomposed into a linear and a nonlinear part as following

$$
\delta(u(t))=u(t)-\phi(u(t))
$$

and there exists a scalar $0<\varepsilon<1$, such that:

$$
\varepsilon u^{T}(t) u(t) \geq \phi^{T}(u(t)) \phi(u(t))
$$

Lemma2 [14] For matrix $\mathrm{R}>0$ and $X^{T}=X$, we have

$$
-X R^{-1} X \leq \rho^{2} R-2 \rho X
$$

Definition 1 A quantizer is called to be logarithmic if the set of quantization levels $\Pi$ can be described

$$
\Pi=\left[ \pm \pi_{i}: \pi_{i}=\rho^{i} \pi_{0}, \pi_{0}>0, i \in \square\right] \cup\{0\}, 0<\rho<1
$$


where $\rho$ is called the quantization density, besides, $\rho=\frac{1-k}{1+k}$, where $0<k<1$. Each of the quantization level corresponds to a segment such that the quantizer maps the whole segment to this quantization level. Precisely, the logarithmic quantization law of $q(\square)$ is defined as follows:

$$
q(w)=\left\{\begin{array}{lr}
v_{i}, \frac{1+\rho}{2} v_{i}<w \leq \frac{1+\rho}{2 \rho} v_{i}, & w>0, \\
0, & w=0, \\
-q(-w), & w<0 .
\end{array}\right.
$$

It can be found from [] and above Definition 1 that $q_{j}(\cdot)(j=1,2, \cdots m)$ can be expressed by $q_{j}\left(x_{j}\right)=\left(1+\Delta_{q_{j}}\left(x_{j}\right)\right) x_{j}$ for certain $\left|\Delta_{q_{j}}\left(x_{j}\right)\right| \leq \varsigma_{q_{j}}(j=1,2, \cdots m) \cdot \varsigma_{q_{j}}(j=1,2, \cdots m)$ are all positive constants. For simplicity, we denote $\Delta_{q_{j}}\left(x_{j}\right)$ as $\Delta_{q_{j}}$. Define

$$
\Delta_{q}=\operatorname{diag}\left\{\Delta_{q_{1}}, \Delta_{q_{2}}, \cdots \Delta_{q_{m}}\right\}
$$

Thus, $q(\cdot)$ can be expressed as

$$
q(x)=\left(I+\Delta_{q}\right) x
$$

Lemma 3 [17] Let $z(t) \in W[a, b)$ and $z(\mathrm{a})=0$. Then, for any $n \times n$ matrix $\mathrm{R}>0$ the following inequality holds:

$$
\int_{a}^{b} z(s) R z(s) d s \leq \frac{4(b-a)^{2}}{\pi^{2}} \int_{a}^{b} \dot{z}(s) R \dot{z}(s) d s
$$

Lemma4(S-procedure)Let $N=N^{\mathrm{T}}, H, E, F_{q}$ be real matrices with appropriate dimensions, for any $F_{q}$ which satisfying $F_{q}^{\mathrm{T}} F_{q} \leq I$. Then the inequality $N+H F E+(H F E)^{\mathrm{T}}<0$ if and only if there exists a positive scalar $\varepsilon$ such that $N+\varepsilon H H^{\mathrm{T}}+\varepsilon^{(-1)} E^{\mathrm{T}} E<0$, or, equivalently,

$$
\left[\begin{array}{ccc}
N & \varepsilon H & E^{\mathrm{T}} \\
\varepsilon H^{\mathrm{T}} & -\varepsilon I & 0 \\
E & 0 & -\varepsilon I
\end{array}\right]<0
$$

\section{Problem Formulation}

\subsection{Sampled-data system}

Consider thesampled-data system scheme as the following:

$$
\left\{\begin{array}{c}
\dot{x}(t)=A x(t)+B_{1} \delta_{u}(u(t)) \\
y(t)=C x(t)
\end{array}\right.
$$


where $x(t) \in \square^{n}$ is the state vector, $u(t) \in \square^{m}$ is the control input vector, $\delta_{u}(u(t))$ are denoted as actuator saturation function; $y(t) \in \square^{p}$ is the measuredoutput vector.

\subsection{Dynamic output-feedback controller}

We denote $t_{k}(k=0,1,2, \cdots)$ as the updating instant time of the Zero-Order-Hold(ZOH) and at the same time denote this updating signal at the sensor $t_{k}-\tau_{m}$. Supposing $t_{k+1}-t_{k} \leq h(k=0,1,2, \cdots)$, where $h$ is a positive scalar which is the largest sampling interval.

Defining $\tau(t)=t-\left(t_{k}-\tau_{m}\right), t_{k} \leq t<t_{k+1}$, Then, the delay $\tau(t)$ satisfies:

$$
\tau_{m} \leq t_{k}-\left(t_{k}-\tau_{m}\right) \leq \tau(t)<t_{k+1}-\left(t_{k}-\tau_{m}\right)<h+\tau_{m}=\tau_{M}
$$

In this paper, we propose to design a stabilizing dynamic output-feedback controller described by:

$$
\left\{\begin{array}{c}
\dot{x_{c}}(t)=A_{c} x_{c}(t)+B_{c} x_{c}(t-\tau(t))+C_{c} q\left(y_{s}(t)\right) \\
u(t)=D_{c} x_{c}(t)
\end{array}\right.
$$

where $x_{c} \in \mathrm{R}^{n_{c}}$ is the controller state vector, $y_{s}(t) \in \mathrm{R}^{p}$ is the controller input. $q\left(y_{s}(t)\right)$ represents thelogarithmicquantization of $y_{s}(t) . A_{c}, B_{c}, C_{c}$ and $D_{c}$ are matrices of appropriate dimensions which are to be determined, $\tau(t)$ is defined as above.

Through the above analysis, we have $y_{s}(t)=y\left(t_{k}\right) t_{k} \leq t<t_{k+1}, k=0,1,2, \cdots$, also we can derive that the sensor output has a form $y_{s}\left(t_{k}\right)=y\left(t_{k}-\tau_{m}\right)$, considering the behavior of the ZOH, we have $q\left(y_{s}(t)\right)=q\left(y\left(t_{k}-\tau_{m}\right)\right), \quad t_{k} \leq t<t_{k+1}, k=0,1,2, \cdots$. By the definition of $\tau(t)$ , we can derive: $y\left(t_{k}-\tau_{m}\right)=y(t-\tau(t))$. Based on the Definition 1,

$$
q\left(y_{s}(\mathrm{t})\right)=q(y(t-\tau(t)))=\left(I+\Delta_{q}\right) y(t-\tau(t))=\left(I+\Delta_{q}\right) C x(t-\tau(t))
$$

Based on Lemma 1, we have:

$$
\delta_{u}(u(t))=u(t)-\phi_{u}(u(t))=D_{c} x_{c}(t)-\phi_{u}(u(t))
$$

and there exists a scalar $0<\varepsilon_{1}<1$ satisfying:

$$
\varepsilon_{1} u^{T}(t) u(t)-\phi_{u}^{T}(u(t)) \phi_{u}(u(t)) \geq 0
$$

\subsection{Augmented system}

From above analysis, substituting (9) and (8) into (6) and (7) respectively, the closed-loop system with dynamic output-feedback control can be described as:

$$
\left\{\begin{array}{c}
\dot{x(t)}=A x(t)+B_{1} D_{c} x_{c}(t)-B_{1} \phi_{u}(u(t)) \\
\dot{x_{c}}(t)=A_{c} x_{c}(t)+B_{c} x_{c}(t-\tau(t))+C_{c}\left(I+\Delta_{q}\right) C x(t-\tau(t))
\end{array}\right.
$$


Set $\xi(t)=\left[\begin{array}{c}x(t) \\ x_{c}(t)\end{array}\right]$, and $\mathrm{A}:=\left[\begin{array}{cc}A & B_{1} D_{c} \\ 0 & A_{c}\end{array}\right], \mathrm{B}:=\left[\begin{array}{cc}0 & 0 \\ C_{c}\left(I+\Delta_{q}\right) C & B_{c}\end{array}\right], \mathrm{C}:=\left[\begin{array}{c}-B_{1} \\ 0\end{array}\right]$

Especially, we denote $\quad E:=\left[\begin{array}{ll}I & 0\end{array}\right], H:=\left[\begin{array}{ll}0 & I\end{array}\right]$

So, (11) can be written as

$$
\dot{\xi}(t)=\mathrm{A} \xi(t)+\mathrm{B} \xi(t-\tau(t))+\mathrm{C} \phi_{u}(u(t))
$$

and the inequality (10) can be described by:

$$
\varepsilon_{1} \xi^{\mathrm{T}}(t) H^{\mathrm{T}} D_{c}^{\mathrm{T}} D_{c} H \xi(t)-\phi_{u}^{\mathrm{T}}(u(t)) \phi_{u}(u(t)) \geq 0
$$

\section{MAIN RESULTS}

\subsection{SUFFICIENT CONDITIONS OF STABILITY}

In this section, we will give asymptotical control stability criteria for system (11) under saturation and quantization condition .

Theorem 1.For given parameters $\tau_{m}$ and $\tau_{M}, \tau_{M} \geq \tau_{m}>0$ and scalars $\varepsilon_{1} \in(0,1)$, the sampled-based closed-loop system (11) with saturation and quantization constraints is asymptotically stable if there exist matrices $P>0, M>0, Q_{i}>0, R_{i}>0(\mathrm{i}=1,2)$ and appropriate matrix $S$ which satisfy: $\left[\begin{array}{cc}R_{2} & S^{\mathrm{T}} \\ S & R_{2}\end{array}\right] \geq 0$

$$
\Pi_{1}=\left[\begin{array}{ccccccccc}
\Pi_{11} & P \mathrm{~B} & R_{1} & 0 & P \mathrm{C} & H^{T} D_{c}^{T} & \tau_{m} \mathrm{~A}^{T} & \tau_{M n} \mathrm{~A}^{T} & \tau_{M n} \mathrm{~A}^{T} \\
* & \Pi_{22} & \Pi_{23} & R_{2}-S^{T} & 0 & 0 & \tau_{m} \mathrm{~B}^{T} & \tau_{M n} \mathrm{~B}^{T} & \tau_{M n} \mathrm{~B}^{T} \\
* & * & \Pi_{33} & S^{T} & 0 & 0 & 0 & 0 & 0 \\
* & * & * & -Q_{2}-R_{2} & 0 & 0 & 0 & 0 & 0 \\
* & * & * & * & -I & 0 & \tau_{m} \mathrm{C}^{T} & \tau_{M n} \mathrm{C}^{T} & \tau_{M n} \mathrm{C}^{T} \\
* & * & * & * & * & -\left(\varepsilon_{1} I\right)^{-1} & 0 & 0 & 0 \\
* & * & * & * & * & * & -R_{1}^{-1} & 0 & 0 \\
* & * & * & * & * & * & * & -R_{2}^{-1} & 0 \\
* & * & * & * & * & * & * & * & -W^{-1}
\end{array}\right]<0
$$

where $\quad \tau_{M m}:=\tau_{M}-\tau_{m} \quad, \quad \Pi_{11}:=P \mathrm{~A}+\mathrm{A}^{T} P+Q_{1}-R_{1} \quad, \quad \Pi_{22}:=-2 R_{2}+S+S^{T}-\frac{\pi^{2}}{4} M$, $\Pi_{23}:=R_{2}-S+\frac{\pi^{2}}{4} M, \Pi_{33}:=Q_{2}-Q_{1}-R_{1}-R_{2}-\frac{\pi^{2}}{4} M$.

Proof: Construct a discontinuous Lyapunov-Krasovskii function for system (11) as

$$
V(t)=V_{1}(t)+V_{2}(t)+V_{3}(t)+V_{4}(t), t \in\left[t_{k}, t_{k+1}\right)
$$

In which $\quad V_{1}(t)=\xi^{\mathrm{T}}(t) P \xi(t), \quad V_{2}(t)=\int_{t-\tau_{m}}^{t} \xi^{\mathrm{T}}(s) Q_{1} \xi(s) d s+\int_{t-\tau_{M}}^{t-\tau_{m}} \xi^{\mathrm{T}}(s) Q_{2} \xi(s) d s$ 
$V_{3}(t)=\tau_{m} \int_{-\tau_{m}}^{0} \int_{t+\theta}^{t} \dot{\xi}^{\mathrm{T}}(s) R_{1} \dot{\xi}(s) d s d \theta+\left(\tau_{M}-\tau_{m}\right) \int_{-\tau_{M}}^{-\tau_{m}} \int_{t+\theta}^{t} \dot{\xi}^{\mathrm{T}}(s) R_{2} \dot{\xi}(s) d s d \theta$

$V_{4}(t)=\left(\tau_{M}-\tau_{m}\right)^{2} \int_{t_{k}-\tau_{m}}^{t} \xi^{\mathrm{T}}(s) M \xi(s) d s-\frac{\pi^{2}}{4} \int_{t_{k}-\tau_{m}}^{t-\tau_{m}}\left[\xi(s)-\xi\left(t_{k}-\tau_{m}\right)\right]^{\mathrm{T}} M\left[\xi(s)-\xi\left(t_{k}-\tau_{m}\right)\right] d s$

$V_{4}(t)$ can be decomposed as a discontinuous term $V_{4 a}(t)$ and a continuous one $V_{4 b}(t)$, that is to say:

$$
V_{4}(t)=V_{4 a}(t)+V_{4 b}(t)
$$

Let:

$$
\begin{gathered}
V_{4 a}(t)=\left(\tau_{M}-\tau_{m}\right)^{2} \int_{t_{k}-\tau_{m}}^{t-\tau_{m}} \xi^{\mathrm{T}}(s) M \xi(s) d s-\frac{\pi^{2}}{4} \int_{t_{k}-\tau_{m}}^{t-\tau_{m}}\left[\xi(s)-\xi\left(t_{k}-\tau_{m}\right)\right]^{\mathrm{T}} M\left[\xi(s)-\xi\left(t_{k}-\tau_{m}\right)\right] d s \\
V_{4 b}(t)=\left(\tau_{M}-\tau_{m}\right)^{2} \int_{t-\tau_{m}}^{t} \xi^{\mathrm{T}}(s) M \xi(s) d s
\end{gathered}
$$

According to Lemma 3, we can see $V_{4 a}(t) \geq 0$, and $V_{4 a}(t)$ vanishes at $t=t_{k}$. Besides, $V_{4 b}(t) \geq 0$. Thus,

$$
\lim _{t \rightarrow t_{k}^{-}}(V(t)) \geq\left(V\left(t_{k}\right)\right)
$$

The time derivative of $\dot{V}(t, \xi(t))$ along the trajectory of system (12) is

$$
\begin{gathered}
\dot{V}(t)=\dot{V}_{1}(t)+\dot{V}_{2}(t)+\dot{V}_{3}(t)+\dot{V}_{4}(t) \\
\dot{V}_{1}(t)=2 \xi^{\mathrm{T}}(t) P \dot{\xi}(t) \\
\dot{V}_{2}(t)=\xi^{\mathrm{T}}(t) Q_{1} \xi(t)-\xi^{\mathrm{T}}\left(t-\tau_{M}\right) Q_{2} \xi\left(t-\tau_{M}\right)+\xi^{\mathrm{T}}\left(t-\tau_{m}\right)\left(Q_{2}-Q_{1}\right) \xi\left(t-\tau_{m}\right) \\
\dot{V}_{3}(t)=-\tau_{m} \int_{t-\tau_{m}}^{t} \dot{\xi}^{\mathrm{T}}(s) R_{1} \dot{\xi}(s) d s-\left(\tau_{M}-\tau_{m}\right) \int_{t-\tau_{M}}^{t-\tau_{m}} \dot{\xi}^{\mathrm{T}}(s) R_{2} \dot{\xi}(s) d s \\
+\dot{\xi}^{\mathrm{T}}(t)\left[\tau_{m}^{2} R_{1}+\left(\tau_{M}-\tau_{m}\right)^{2} R_{2}\right] \dot{\xi}(t) \\
\dot{V}_{4}(t)=\left(\tau_{M}-\tau_{m}\right)^{2} \dot{\xi}^{\mathrm{T}}(t) M \dot{\xi}(t)-\frac{\pi^{2}}{4}\left[\xi\left(t-\tau_{m}\right)-\xi(t-\tau(t))\right]^{T} M\left[\xi\left(t-\tau_{m}\right)-\xi(t-\tau(t))\right]
\end{gathered}
$$

Using Jensen inequality, we can obtain

$$
-\tau_{m} \int_{t-\tau_{m}}^{t} \xi^{\mathrm{T}}(s) R_{1} \xi(s) d s \leq-\left[\xi(t)-\xi\left(t-\tau_{m}\right)\right]^{\mathrm{T}} R_{1}\left[\xi(t)-\xi\left(t-\tau_{m}\right)\right]
$$

When

$$
\left[\begin{array}{ll}
R_{2} & S^{\mathrm{T}} \\
S & R_{2}
\end{array}\right] \geq 0
$$

the following can be derived:

$$
\begin{aligned}
-\left(\tau_{M}-\tau_{m}\right) \int_{t-\tau_{M}}^{t-\tau_{m}} \dot{\xi}^{\mathrm{T}}(s) R_{2} \dot{\xi}(s) d s & \leq-\left[\xi\left(t-\tau_{m}\right)-\xi(t-\tau(t))\right]^{\mathrm{T}} R_{2}\left[\xi\left(t-\tau_{m}\right)-\xi(t-\tau(t))\right] \\
& -\left[\xi(t-\tau(t))-\xi\left(t-\tau_{M}\right)\right]^{\mathrm{T}} R_{2}\left[\xi(t-\tau(t))-\xi\left(t-\tau_{M}\right)\right] \\
& -2\left[\xi\left(t-\tau_{m}\right)-\xi(t-\tau(t))\right]^{\mathrm{T}} S^{\mathrm{T}}\left[\xi(t-\tau(t))-\xi\left(t-\tau_{M}\right)\right]
\end{aligned}
$$


Substituting (17)in (16) and combining (18) and (19) into consideration, and adding saturation condition (13), we have

$$
\dot{V}(t) \leq \eta^{T}(t) \Pi_{1} \eta(t)
$$

Where $\eta^{T}(t)=\left[\xi^{T}(t), \xi^{T}(t-\tau(t)), \xi^{T}\left(t-\tau_{m}\right), \xi^{T}\left(t-\tau_{M}\right), \phi_{u}^{T}(u(t))\right]$

If condition (14) is satisfied, it can be seen that the closed loop system (11) is asymptotically stable. This completes the proof.

\subsection{Explicit form of sufficient conditions}

In the following theorem we will give the explicit form of the desired DOFC parameter.

Theorem 2.Based on condition and result of theorem 1, for given parameters $\tau_{m}$ and $\tau_{M}$, $\tau_{M} \geq \tau_{m}>0$ and scalars $\varepsilon_{i} \in(0,1)(i=1,2)$,the sampled-based closed-loop system(11) with saturation and quantization constraints is asymptotically stable if there exist matrices $P>0, M>0, Q_{i}>0, R_{i}>0(\mathrm{i}=1,2)$ and appropriate matrix $S$ which satisfy:

$$
\Pi_{2}=\left[\begin{array}{ccc}
\bar{\Pi} & \Gamma_{1}^{\mathrm{T}} & \varepsilon_{2} \Gamma_{2}^{\mathrm{T}} \\
* & -\varepsilon_{2} I & 0 \\
* & * & -\varepsilon_{2} I
\end{array}\right]<0
$$

where $\Gamma_{1}=[\left(P \bar{C}_{c}\right)^{\mathrm{T}}, \overbrace{0, \cdots 0}^{5}, \tau_{m} \bar{C}_{c}^{\mathrm{T}},\left(\tau_{M}-\tau_{m}\right) \bar{C}_{c}^{\mathrm{T}},\left(\tau_{M}-\tau_{n}\right) \bar{C}_{c}^{\mathrm{T}}], \Gamma_{2}=[0, \bar{C}, \overbrace{0, \cdots 0}^{7}]$,

$$
\overline{\Pi_{1}}=\left[\begin{array}{ccccccccc}
\Pi_{11} & P \mathrm{~B}_{1} & R_{1} & 0 & P \mathrm{C} & H^{T} D_{c}^{T} & \tau_{m} \mathrm{~A}^{T} & \tau_{M n} \mathrm{~A}^{T} & \tau_{M n} \mathrm{~A}^{T} \\
* & \Pi_{22} & \Pi_{23} & R_{2}-S^{T} & 0 & 0 & \tau_{m} \mathrm{~B}_{1}^{T} & \tau_{M n} \mathrm{~B}_{1}^{T} & \tau_{M n} \mathrm{~B}_{1}^{T} \\
* & * & \Pi_{33} & S^{T} & 0 & 0 & 0 & 0 & 0 \\
* & * & * & -Q_{2}-R_{2} & 0 & 0 & 0 & 0 & 0 \\
* & * & * & * & -I & 0 & \tau_{m} \mathrm{C}^{T} & \tau_{M n} \mathrm{C}^{T} & \tau_{M n} \mathrm{C}^{T} \\
* & * & * & * & * & -\left(\varepsilon_{1} I\right)^{-1} & 0 & 0 & 0 \\
* & * & * & * & * & * & -R_{1}^{-1} & 0 & 0 \\
* & * & * & * & * & * & * & -R_{2}^{-1} & 0 \\
* & * & * & * & * & * & * & * & -W^{-1}
\end{array}\right]
$$

Where $\Pi_{11}, \Pi_{22}, \Pi_{22}, \Pi_{22}$ is the same as given in Theorem 1, and $\mathrm{B}_{1}=\left[\begin{array}{cc}0 & 0 \\ C_{c} C & B_{c}\end{array}\right]$.

Proof: First, we decompose: $\mathrm{B}=\mathrm{B}_{1}+\mathrm{B}_{0}$

$$
\begin{gathered}
\mathrm{B}_{1}=\left[\begin{array}{cc}
0 & 0 \\
C_{c} C & B_{c}
\end{array}\right], \mathrm{B}_{0}=\left[\begin{array}{cc}
0 & 0 \\
C_{c} \Delta_{q} C & 0
\end{array}\right]=\left[\begin{array}{c}
0 \\
C_{c}
\end{array}\right] F_{q}\left[\begin{array}{ll}
\bar{\Delta} C & 0
\end{array}\right] \\
\text { and in which } \Delta_{q}=F_{q} \bar{\Delta}, \quad F_{q}^{\mathrm{T}} F_{q} \leq I
\end{gathered}
$$




$$
\text { and simultaneously, we set } \bar{C}_{c}=\left[\begin{array}{c}
0 \\
C_{c}
\end{array}\right], \quad \bar{C}=\left[\begin{array}{ll}
\bar{\Delta} C & 0
\end{array}\right]
$$

$$
\text { Then, we can derive } \mathrm{B}_{0}=\bar{C}_{c} F_{q} \bar{C}
$$

Thus, we can rewrite $\Pi_{1}$ as the following form:

$$
\Pi_{1}=\overline{\Pi_{1}}+\Gamma_{1}^{\mathrm{T}} F_{q} \Gamma_{2}+\Gamma_{2}^{\mathrm{T}} F_{q}^{\mathrm{T}} \Gamma_{1}
$$

By utilizing Lemma 4,(26) is equivalent that there exist positive scalars $\varepsilon_{2}$ satisfying

$$
\Pi_{1}=\bar{\Pi}_{1}+\varepsilon_{2}^{-1} \Gamma_{1}^{\mathrm{T}} \Gamma_{1}+\varepsilon_{2} \Gamma_{2}^{\mathrm{T}} \Gamma_{2}
$$

And based on Schur complement, (21) is easy to obtain and the proof is completed.

\subsection{Dynamic output-feedback controller}

The following theorem presents the explicit parameters of the desired dynamic output feedback controller based on theorem 1 and theorem 2 .

Theorem 3.Based on condition and result of theorem 1and theorem 2, for given parameters $\tau_{m}$ and $\tau_{M}, \tau_{M} \geq \tau_{m}>0$ and scalars $\varepsilon_{i} \in(0,1)(i=1,2)$, the augmented system (12) is asymptotically stable there with system (11) is asymptotically stable if there exist matrices $P>0, M>0, Q_{i}>0, R_{i}>0(\mathrm{i}=1,2)$ and appropriate matrix $S$ which satisfy:

$$
\begin{aligned}
& {\left[\begin{array}{cc}
\tilde{R}_{2} & \tilde{S}^{T} \\
\tilde{S} & \tilde{R}_{2}
\end{array}\right] \geq 0, \quad Z:=\left[\begin{array}{cc}
X & I \\
* & P_{11}
\end{array}\right]>0} \\
& \text { and } \Pi_{3}=\left[\begin{array}{ccc}
\Pi & F_{1}^{\mathrm{T}} & \varepsilon_{2} \vec{F}_{2}^{\mathrm{T}} \\
* & -\varepsilon_{2} I & 0 \\
* & * & -\varepsilon_{2} I
\end{array}\right]<0
\end{aligned}
$$

where $\mathrm{F}_{1}=[\varphi_{5}{ }^{\mathrm{T}}, \overbrace{0, \cdots 0}^{5}, \tau_{m} \varphi_{5}{ }^{\mathrm{T}},\left(\tau_{M}-\tau_{m}\right) \varphi_{5}^{\mathrm{T}},\left(\tau_{M}-\tau_{n}\right) \varphi_{5}^{\mathrm{T}}], \mathrm{F}_{2}=[0, \varphi_{6}, \overbrace{0, \cdots 0}^{7}]$,

$$
\bar{\Pi}_{1}=\left[\begin{array}{ccccccccc}
\mathrm{H}_{11} & \varphi_{2} & R_{1} & 0 & \varphi_{3} & \varphi_{4} & \tau_{m} \varphi_{1}^{T} & \tau_{M n} \varphi_{1}^{T} & \tau_{M n} \varphi_{1}^{T} \\
* & \mathrm{H}_{22} & \mathrm{H}_{23} & R_{2}-S^{T} & 0 & 0 & \tau_{m} \varphi_{2}^{T} & \tau_{M n} \varphi_{2}^{T} & \tau_{M n} \varphi_{2}^{T} \\
* & * & \mathrm{H}_{33} & S^{T} & 0 & 0 & 0 & 0 & 0 \\
* & * & * & -Q_{2}-R_{2} & 0 & 0 & 0 & 0 & 0 \\
* & * & * & * & -I & 0 & \tau_{m} \varphi_{3}^{T} & \tau_{M n} \varphi_{3}^{T} & \tau_{M n} \varphi_{3}^{T} \\
* & * & * & * & * & -\left(\varepsilon_{1} I\right)^{-1} & 0 & 0 & 0 \\
* & * & * & * & * & * & R_{1}-2 Z & 0 & 0 \\
* & * & * & * & * & * & * & R_{2}-2 Z & 0 \\
* & * & * & * & * & * & * & * & W-2 Z
\end{array}\right]
$$


in which $\mathrm{H}_{11}=\varphi_{1}+\varphi_{1}^{T}+Q_{1}-R_{1}, \Pi_{22}=-2 R_{2}+S+S^{T}-\frac{\pi^{2}}{4} M, \Pi_{23}=R_{2}-S+\frac{\pi^{2}}{4} M$, $\Pi_{33}:=Q_{2}-Q_{1}-R_{1}-R_{2}-\frac{\pi^{2}}{4} M$

and $\varphi_{1}=\left[\begin{array}{cc}A X+B_{1} W_{1} & A \\ W_{4} & P_{11} A\end{array}\right], \varphi_{2}=\left[\begin{array}{cc}0 & 0 \\ W_{3} & W_{2} C\end{array}\right], \varphi_{3}=\left[\begin{array}{c}-B_{1} \\ -P_{11}^{T} B_{1}\end{array}\right]$,

$$
\varphi_{4}=\left[\begin{array}{c}
W_{1}^{T} \\
0
\end{array}\right], \quad \varphi_{5}=\left[\begin{array}{c}
0 \\
W_{2}
\end{array}\right], \quad \varphi_{6}=\left[\begin{array}{c}
X C^{T} \bar{\Delta}^{T} \\
C^{T} \bar{\Delta}^{T}
\end{array}\right]
$$

Proof: Since $P>0$, thus the matrix $P$ is nonsingular, then we partition $P$ as

$$
P=\left[\begin{array}{cc}
P_{11} & P_{12} \\
* & P_{22}
\end{array}\right]
$$

Where $P_{11}, P_{12}, P_{22} \in \mathrm{R}^{n \times n}$, what's more, it is easy to see $P_{11}>0$ and $P_{22}>0$. Without loss of generality, suppose $P_{12}$ is nonsingular square matrix. Define the matrix $Z$ which is satisfied $Z>0$, where $Z$ is defined. The relation of $P$ and $Z$ is

$$
\left[\begin{array}{cc}
I & P_{11} \\
0 & P_{12}^{T}
\end{array}\right]=P\left[\begin{array}{cc}
X & P_{12} \\
P_{12}^{-1}(I-P 11 X) & 0
\end{array}\right]
$$

Let

$$
L_{1}=\left[\begin{array}{cc}
X & P_{12} \\
P_{12}^{-1}\left(I-P_{11} X\right) & 0
\end{array}\right] L_{2}=\left[\begin{array}{cc}
I & P_{11} \\
0 & P_{12}^{T}
\end{array}\right]
$$

It is easy to know $L_{1}$ and $L_{2}$ are both non-singular.

Define $\mathrm{L}:=\operatorname{diag}\left\{L_{1}, L_{1}, L_{1}, L_{1}, I, I, L_{2}, L_{2}, L_{2}, I, I\right\}$. Performing congruence transformation to the matrix inequality (21), we get

$$
\Pi_{3}=\mathrm{L}^{T} \Pi_{2} \mathrm{~L}
$$

Denote $\tilde{S}=L_{1}^{T} S L_{1}, \tilde{M}=L_{1}^{T} M L_{1}, \tilde{Q}_{i}=L_{1}^{T} Q_{i} L_{1}, \tilde{R}_{i}=L_{1}^{T} R_{i} L_{1}(i=1,2)$. According to the denotation $\tilde{R}_{i}=L_{1}^{T} R_{i} L_{1}(i=1,2)$, we can derive

$$
R_{i}^{-1}=L_{1} \tilde{R}_{i}^{-1} L_{1}^{T}
$$

In the computational process, we can get the item $-L_{2}{ }^{T} R_{i}^{-1} L_{2}$, substituting (22) in it, we can obtain

$$
-L_{2}^{T} R_{i}^{-1} L_{2}=-L_{2}^{T} L_{1} \tilde{R}_{i}^{-1} L_{1}^{T} L_{2}=-Z^{T} \tilde{R}_{i}^{-1} Z
$$

By using Lemma 2, we have

$$
-Z^{T} \tilde{R}_{i}^{-1} Z \leq \tilde{R}_{i}-2 Z
$$

Similarly, we can easily obtain 


$$
-Z^{T} \tilde{M}^{-1} Z \leq \tilde{M}-2 Z
$$

and we define $W_{i}(\mathrm{i}=1,2,3,4)$ as

$$
\left\{\begin{array}{l}
W_{1}:=D_{c} P_{12}^{-1}\left(I-P_{11} X\right), W_{2}:=P_{12} C_{c} \\
W_{3}:=W_{2} C X+P_{12} B_{c} P_{12}^{-1}\left(I-P_{11} X\right) \\
W_{4}:=P_{11}^{T} A X+P_{11}^{T} B_{1} W_{1}+P_{12} A_{c} P_{12}^{-1}\left(I-P_{11} X\right)
\end{array}\right.
$$

It can be seen that all the process of proof is reversible, therefore, theorem 3 can also deduce Theorem 1 through Theorem 2, that is to say, the dynamic output feedback controller is:

$$
\left\{\begin{array}{l}
A_{c}=P_{12}^{-1}\left(W_{4}-P_{11}^{T} A X-P_{11}^{T} B_{1} W_{1}\right)\left(I-P_{11} X\right)^{-1} P_{12} \\
B_{c}=P_{12}^{-1}\left(W_{3}-W_{2} C X\right)\left(I-P_{11} X\right)^{-1} P_{12} \\
C_{c}=P_{12}^{-1} W_{2}, D_{c}=W_{1}\left(I-P_{11} X\right)^{-1} P_{12}
\end{array}\right.
$$

Remark 1: Though, the matrix of $P_{12}$ is unknown, we can see the DOFC in (7) is equivalent to the following DOFC in (41) by transforming $x_{c}(t)=P_{12}^{-1} \tilde{x}_{c}(t)$ and setting

$$
\left(\tilde{A}_{c}, \tilde{B}_{c}, \tilde{C}_{c}, \tilde{D}_{c}\right):=\left(P_{12} A_{c} P_{12}^{-1}, P_{12} B_{c} P_{12}^{-1}, P_{12} C_{c}, D_{c} P_{12}^{-1}\right) \text {, where } \tilde{A}_{c}, \tilde{B}_{c}, \tilde{C}_{c}, \tilde{D}_{c} \text { can be described as: }
$$

$$
\left\{\begin{array}{l}
\tilde{A}_{c}=\left(W_{4}-P_{11}^{T} A X-P_{11}^{T} B_{1} W_{1}\right)\left(I-P_{11} X\right)^{-1} \\
\tilde{B}_{c}=\left(W_{3}-W_{2} C X\right)\left(I-P_{11} X\right)^{-1} \\
\tilde{C}_{c}=W_{2} \\
\tilde{D}_{c}=W_{1}\left(I-P_{11} X\right)^{-1}
\end{array}\right.
$$

\section{NUMERICAL EXAMPLE}

In this section, a numerical example is presented to show the validity of the control approach for the sampled-data system with actuator saturation using quantized measurements.

Consider the system (6) described as:

$$
\left\{\begin{array}{c}
x(t)=\left[\begin{array}{ccc}
-2 & -0.1 & 0 \\
-0.1 & 0 & 0.2 \\
0 & -0.5 & 0
\end{array}\right] x(t)+\left[\begin{array}{c}
0.1 \\
0.02 \\
0.1
\end{array}\right] \delta_{u}(u(t)) \\
y(t)=\left[\begin{array}{lll}
1 & 0 & 0 \\
0 & 2 & 0
\end{array}\right] x(t)
\end{array}\right.
$$

In the following, our purpose is to design the DOFC in form (41)for the system (42), by setting $\varepsilon_{1}=0.2, \quad \varepsilon_{2}=0.6, \quad k=0.7, \quad \tau_{m}=0.02, \quad \tau_{M}=0.6$, the sampling interval $h=0.05$. Based on the Theorem 3 and Remark 1, we can obtain the corresponding DOFC parameters as:

$$
\tilde{A}_{c}=\left[\begin{array}{ccc}
-2.3132 & -21.3322 & -35.7622 \\
-0.0884 & -0.8822 & -1.1529 \\
0.0772 & -0.1137 & 0.0962
\end{array}\right], \quad \tilde{B}_{c}=\left[\begin{array}{rrr}
-0.0013 & 0.0172 & -0.0085 \\
0.0055 & -0.1918 & -0.0667 \\
-0.0005 & 0.0424 & 0.0394
\end{array}\right]
$$




$$
\tilde{C}_{c}=\left[\begin{array}{rr}
-0.0849 & 0.2176 \\
0.0255 & -1.5719 \\
-0.0318 & 0.1740
\end{array}\right], \quad \quad \tilde{D}_{c}=\left[\begin{array}{lll}
0.0124 & 1.0490 & 1.6772
\end{array}\right]
$$

When the initial condition is chosen as $x(0)=\left[\begin{array}{lll}2.6 & -1.5 & 1.8\end{array}\right]^{T}$, the responses of the state $x$ and the saturation control signal $\delta_{u}(u(t))$ are shown in Figures 1 and Figures 2, respectively. It can be seen that the closed-loop system is asymptotically stable. Simulation results indicate the effectiveness of the proposed control technology.

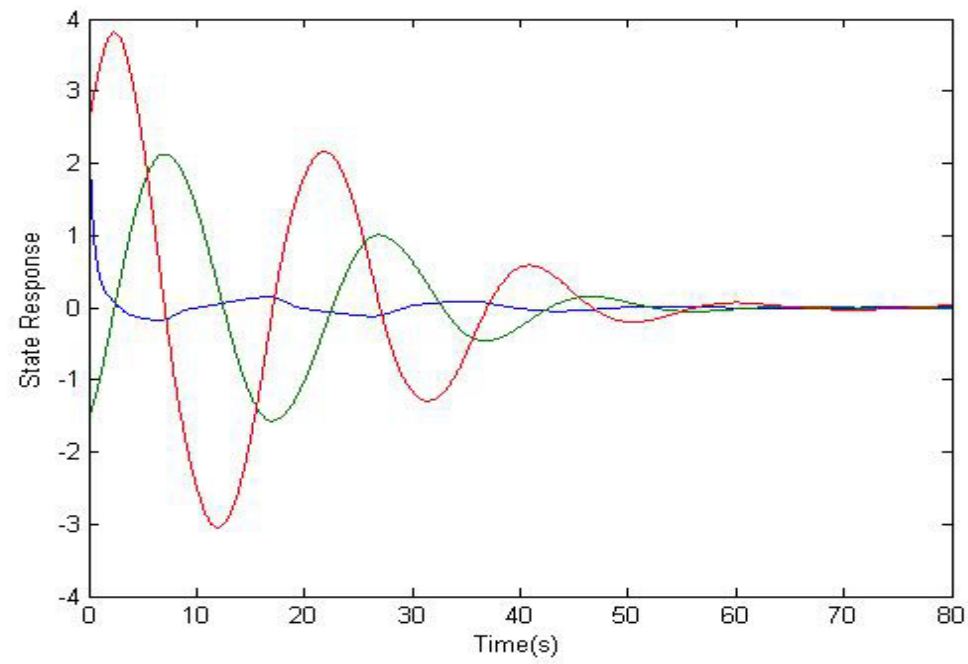

Figure 1. The state responses under DOFC

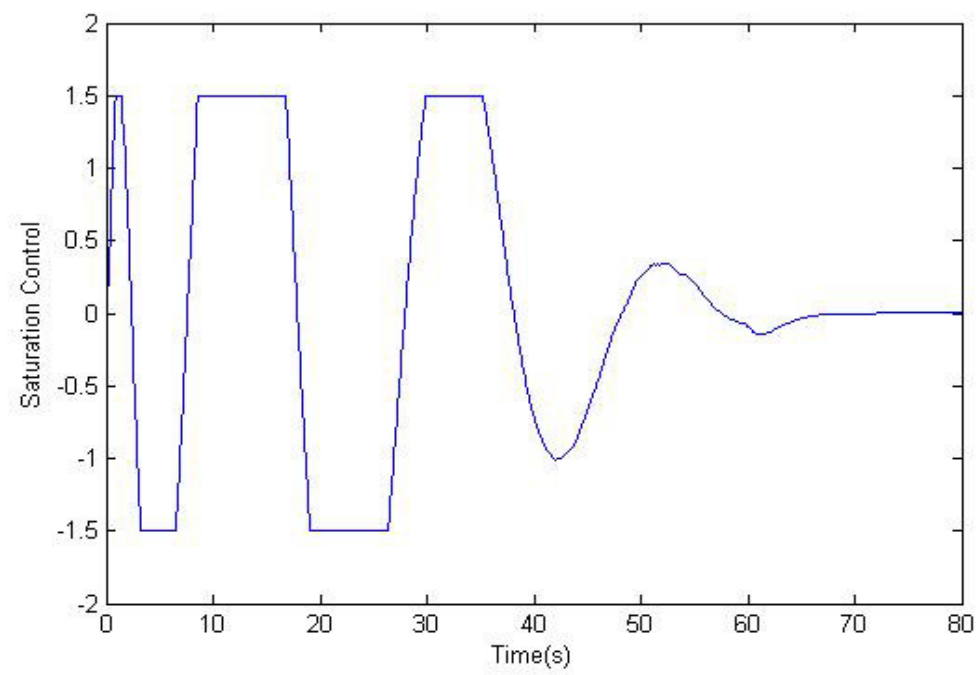

Figure 2. Output of saturation actuator 


\section{Conclusions}

In this paper, using discontinuous Lyapunov functional technique, we study the dynamic output feedback control of sampled-data system with saturation and quantization. A time-varying delay model with nonlinear items is represented as the closed-loop system, and a novel stability criterion is established by discontinuous Lyapunov functional approach. Sufficient conditions for asymptotic stability are derived employing linear matrix inequality approach and the corresponding matrix of DOFC are both designed. A numerical example shows that the results obtained in this paper are very practicable in analyzing the stability of the sampled data control system.

\section{ACKNOWLEDGEMENTS}

This work was supported by the Natural Science Foundation of China (NSFC) (No. 61273119, No. 61305011), Natural Science Foundation of Jiangsu Province of China (No. BK20150793), Plan of Policy Guidance in Jiangsu Province(No. BY2015004-11), and Jiangsu Agricultural Science and Technology Independent Innovation Fund Project (No. CX(15)1051).

\section{REFERENCES}

[1] W. Zhang. M. Branicky \& S. Phillips, (2001) "Stability of networked control systems”, IEEE Control System Magazine, Vol. 21, No. 1, pp84-99.

[2] E. Fridman, (2010) “A refined input delay approach to sampled-data control”, Automatica, Vol. 46, No. 2, pp421-427.

[3] H. Wang. Y, Ying \& R. Lu, (2016) "Network-based H-infinity control for singular systems with event-triggered sampling scheme", Information Sciences, Vol. 329, No. 1, pp540-551.

[4] D. Yue, E. Tian\&Q. Han, (2013) "A delay system method for designing event-triggered controllers of networked control systems", IEEE Transactions on Automatic Control, Vol. 58, No. 2, pp475-481.

[5] F. Yang \& Y. Li, (2009) "Set-membership filtering for systems with sensor saturation", Automatica, Vol. 45, No. 8, pp1896-1920.

[6] Z. Wang,B. Shen \& X. Liu, (2012) “ filtering with randomly occurring sensor saturations and missing measurements", Automatica, Vol. 48, No. 3, pp556-562.

[7] Z. Wang, W. Daniel, H. Daniel \& H. Gao, (2010) “ finite-horizon control for a class of stochastic nonlinear time-varying systems subject to sensor and actuator saturations", IEEE Transactions on Automatic Control, Vol. 55, No. 7, pp1716-1722.

[8] Y. Wei, M. Liu \& P. Shi, (2012) “ filtering for nonlinear stochastic systems with sensor saturation, quantization and random packet losses”, Signal Processing, Vol. 92, No. 6, pp1387-1396.

[9] T. Shi, H. Su \& J. Chu, (2013) "Reliable filtering for linear systems with sensor saturation", Journal of Control Theory and Applications, Vol. 11, No. 1, pp80-85.

[10] Z. Gu, S. Fei, Y. Zhao \& E. Tian, (2014) "Robust control of automotive active seat-suspension system subject to actuator saturation", Journal of Dynamic Systems, Measurement, and Control, Vol. 136, No. 4, pp041022(1)-041022(7).

[11] G. Garcia, S. Tarbouriech \& M. da Silva, (2007) "Dynamic output controller design for linear systems with actuator and sensor saturation”, IEEE American Control Conference, pp5834-5839.

[12] C. Peng \& M. Fei, (2013) "An improved result on the stability of uncertain T-S fuzzy systems with interval time-varying delay”, Fuzzy Sets and Systems, Vol. 212, No. 1, pp97-109. 
[13] L. Sun, Y. Wang \& G. Feng, (2015) "Control design for a class of affine nonlinear descriptor systems with actuator saturation", Transactions on Automatic Control, Vol. 60, No. 8, pp2195-2200.

[14] J. Xiong \& L. James, (2009) "Stabilization of networked control systems with a logic ZOH", IEEE Transactions on Automatic Control, Vol. 54, No. 2, pp358-363.

[15] K. Liu \& E. Fridman, (2012) "Wirtinger's inequality and Lyapunov-based sampled-data stabilization", Automatica, Vol. 48, No. 1, pp102-108.

[16] X. Liu, W. Yu, J. Cao \& S. Chen, (2015) "Discontinuous Lyapunov approach to state estimation and filtering of jumped systems with sampled-data”, Neural Networks, Vol. 68, No. 1, pp12-22.

[17] K. Liu, V. Suplin \& E. Fridman, (2010) "Stability of linear systems with general sawtooth delay", IMA Journal of Mathematical Control and Information, Vol. 27, No. 4, pp419-436.

[18] S. Lakshmanan, J. Park, R. Rakkiyappan \&H. Jung, (2013) "State estimator for neural networks with sampled data using discontinuous Lyapunov functional approach", Nonlinear Dynamics, Vol. 73, No. 1-2, pp509-520.

[19] Z. Chen, K. Shi \& S. Zhong, (2016) "New synchronization criteria for complex delayed dynamical networks with sampled-data feedback control”, ISA Transactions, Vol. 63, pp154-169.

[20] E. Tian, W. Wong, D. Yue \& T Yang, (2015) “ Filtering for Discrete-Time Switched Systems with Known Sojourn Probabilities”, IEEE Transactions on Automatic Control, Vol. 60, No. 9, pp2446-2451.

[21] E. Tian \& D. Yue, (2015) "Decentralized control of network-based interconnected systems: A state-dependent triggering method", International Journal of Robust and Nonlinear control, Vol. 25, No. 8, pp1126-1144.

[22] C. Peng \& Q. Han, (2016) "On designing a novel self-triggered sampling scheme for networked control systems with network-induced delays and data dropouts”, IEEE Transactions on Industrial Electronics, Vol. 63, No. 2, pp1239-1248.

[23] C. Peng, Y. Song, X. Xie, M. Zhao \& M. Fei, (2016) “An event-triggered output tracking control for wireless networked control systems with communication delays and data dropouts", IET Control Theory and Applications, Vol. 10, No. 17, pp2195-2203.

[24] E. Tian, D. Yue \& C. Peng, (2008) "Quantized output feedback control for networked control systems", Information sciences, Vol. 178, No. 12, pp2734-2749.

[25] M, Fu\& L Xie, (2005) "The sector bound approach to quantized feedback control", IEEE Transactions on Automatic control, Vol. 50, No. 11, pp1698-1711.

[26] H, Gao \& TChen, (2008) "A new approach to quantized feedback control systems", Automatica, Vol. 44, No. 2, pp534-542.

[27] S. Yun, J. Yun \& PPark, (2010) "Dynamic output-feedback guaranteed cost control for linear systems with uniform input quantization”, Nonlinear Dynamics, Vol. 62, No. 1, pp95-104. 


\section{AUTHORS}

\section{Liuwen Li}

received the M.S.degree in College of Science from Nanjing University of Aeronautics and Astronautics, Nanjing, China, in 2008. She is currently a PhD student of School of Automation Southeast University, Nanjing, China .Her current research includes time-delay systems and networked control systems, etc.

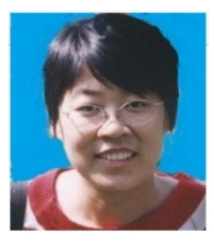

\section{WenlinZou}

received his Ph.D. degree in engineering from School of Automation, Southeast University, Nanjing, China, in 2014. Presently, he is doing postdoctoral research at School of Computer Science and Engineering, Nanjing University of Science and Technology, Nanjing, China. His current research interests include neural networks, time-delay systems, networked control systems, etc.

\section{Shumin Fei}

received the Ph.D. degree from Beijing University of Aeronautics and Astronautics in 1995, China. From 1995 to 1997, he was doing postdoctoral research at Research Institute of Automation, Southeast University, China. Presently, he is a professor and doctor adviser at Southeast University in China. He has published more than 70 journal papers and his current research interests include nonlinear systems, time-delay system, complex systems, and so on.
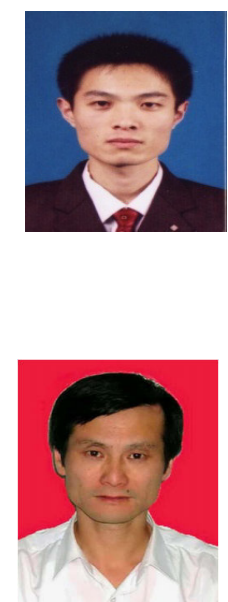